IFUP-TH 2001/36

\title{
RENORMALIZATION-GROUP FLOW IN THE 3D GEORGI-GLASHOW MODEL
}

\author{
DMITRI ANTONOV \\ INFN-Sezione di Pisa, Universitá degli studi di Pisa, Dipartimento di Fisica, Via \\ Buonarroti, 2 - Ed. B - I-56127 Pisa, Italy \\ and \\ Institute of Theoretical and Experimental Physics, B. Cheremushkinskaya 25, \\ $R U$-117 218 Moscow, Russia \\ E-mail: antonov@df.unipi.it
}

\begin{abstract}
The renormalization-group ( $\mathrm{RG})$ flow in the finite-temperature (2+1)-dimensional Georgi-Glashow model is explored. This is done in the limit when the squared electric coupling constant is much larger than the mass of the Higgs field. The novel equation describing the evolution of the Higgs mass is derived and integrated along the separatrices of the RG flow in the limit when the original theory reduces to the 2D XY model. In particular, it is checked that in the vicinity of the phasetransition point, there exists a range of parameters allowing to the Higgs mass evolved along some of the separatrices to remain much smaller than the squared electric coupling constant.
\end{abstract}

\section{The model}

$(2+1)$ D Georgi-Glashow model is known to be the famous example of a theory allowing for an analytical description of confinement 1 . However, the finitetemperature effects in this theory were addressed only recently. Namely, first in ref. 2 the phase transition associated with the binding of monopoles into molecules has been studied and then in ref. $\mathrm{B}$, there has been explored another phase transition corresponding to the deconfinement of charged W-bosons. In this talk, we shall concentrate ourselves at the first of these two phase transitions, but account also for the effects brought about by the Higgs field. In this way, we shall follow the analysis performed in ref. 6 .

The Euclidean action of the $(2+1)$ D Georgi-Glashow model has the following form

$$
S=\int d^{3} x\left[\frac{1}{4 g^{2}}\left(F_{\mu \nu}^{a}\right)^{2}+\frac{1}{2}\left(D_{\mu} \Phi^{a}\right)^{2}+\frac{\lambda}{4}\left(\left(\Phi^{a}\right)^{2}-\eta^{2}\right)^{2}\right],
$$

where the Higgs field $\Phi^{a}$ transforms by the adjoint representation, and

contribution: submitted to World Scientific on December 28, 2013 


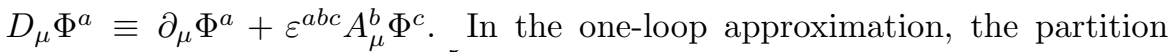
function of this theory reads 0

$$
\begin{gathered}
\mathcal{Z}=1+\sum_{N=1}^{\infty} \frac{\zeta^{N}}{N !}\left[\prod_{i=1}^{N} \int d^{3} z_{i} \sum_{q_{i}= \pm 1}\right] \times \\
\times \exp \left\{-\frac{g_{m}^{2}}{2}\left[\int d^{3} x d^{3} y \rho(\mathbf{x}) D_{0}(\mathbf{x}-\mathbf{y}) \rho(\mathbf{y})-\sum_{\substack{a, b=1 \\
a \neq b}}^{N} D_{m}\left(\mathbf{z}_{a}-\mathbf{z}_{b}\right)\right]\right\} .
\end{gathered}
$$

Here, $g_{m}$ is the magnetic coupling constant of dimensionality [length $]^{1 / 2}$ related to the electric one $g$ according to the equation $g g_{m}=4 \pi, \rho(\mathbf{x})=$ $\sum_{a=1}^{N} q_{a} \delta\left(\mathbf{x}-\mathbf{z}_{a}\right)$ is the density of monopole plasma with $q_{a}$ 's standing for the monopole charges in the units of $g_{m}$. Next, in Eq. (2), $m=\eta \sqrt{2 \lambda}$ is the mass of the Higgs boson and

$$
\zeta=\frac{m_{W}^{7 / 2}}{g} \delta\left(\frac{\lambda}{g^{2}}\right) \mathrm{e}^{-\left(4 \pi / g^{2}\right) m_{W} \epsilon\left(\lambda / g^{2}\right)}
$$

is the statistical weight of a single monopole (else called fugacity) with $m_{W}=$ $g \eta$ being the mass of the $W$-boson. Here, $\epsilon$ is a slowly varying function equal to unity at the origin ( $j e$. in the Bogomolny-Prasad-Sommerfield limit 6 ) and $1.787 \ldots$ at infinity $\mathrm{Q}$, whereas the function $\delta$ is determined by the loop corrections. Finally, in eq. (2), $D_{0}(\mathbf{x}) \equiv 1 /(4 \pi|\mathbf{x}|)$ is the Coulomb propagator, and $D_{m}(\mathbf{x}) \equiv \mathrm{e}^{-m|\mathbf{x}|} /(4 \pi|\mathbf{x}|)$ is the propagator of the Higgs boson.

Notice that as it follows from eq. (2), in the Bogomolny-PrasadSommerfield limit, the interaction of two monopoles doubles for opposite and vanishes for equal charges. As far as the opposite limit, $m \rightarrow \infty$, is concerned, we apparently arrive there at the standard compact-QED result 1 .

The effective field theory describing the grand fanonical partition function (2) can easily be obtained and its action reads

$$
S=\int d^{3} x\left[\frac{1}{2}(\nabla \chi)^{2}+\frac{1}{2}(\nabla \psi)^{2}+\frac{m^{2}}{2} \psi^{2}-2 \zeta \mathrm{e}^{g_{m} \psi} \cos \left(g_{m} \chi\right)\right],
$$

where $\chi$ is the dual photon field, whereas the field $\psi$ is an additional one. The latter field can be integrated out in the limit $g \gg \sqrt{m}$. It can be shown $\mathrm{\theta}$ that in this limit, the exponent in the last term on the r.h.s. of eq. (4) can 
be approximated by the terms not higher than the linear one. (Note that the above inequality is implied only in the polynomial and not in the exponential sense.)

In such a limit, Gaussian integration over the field $\psi$ yields the following action of the dual photon field:

$$
\begin{gathered}
S=\int d^{3} x\left[\frac{1}{2}(\nabla \chi)^{2}-2 \zeta \cos \left(g_{m} \chi\right)\right]- \\
-2\left(g_{m} \zeta\right)^{2} \int d^{3} x d^{3} y \cos \left(g_{m} \chi(\mathbf{x})\right) D_{m}(\mathbf{x}-\mathbf{y}) \cos \left(g_{m} \chi(\mathbf{y})\right) .
\end{gathered}
$$

The last term here represents the correction to the standard result 1 . It stems from the fact that the mass of the Higgs field was considered to be not infinitely large compared to the standard Debye mass of the dual photon, $m_{D}=g_{m} \sqrt{2 \zeta}$. The respective correction to $m_{D}$ is positive, and the square of the full mass reads: $M^{2}=m_{D}^{2}\left(1+\frac{m_{D}^{2}}{m^{2}}\right)$. Clearly, this result is valid at $m_{D} \ll m$ and reproduces $m_{D}^{2}$ in the limit $m \rightarrow \infty$.

Another relation between the dimensionful parameters in the model (1), we shall adapt for our analysis, is $g \ll \eta$. [Clearly, this inequality parallels the requirement that $\eta$ should be large enough to ensure the spontaneous symmetry breaking from $S U(2)$ to $U(1)$.] In particular, from this relation and the inequality $g \gg \sqrt{m}$ we immediately obtain: $\frac{\lambda}{g^{2}} \sim\left(\frac{m}{m_{W}}\right)^{2} \ll\left(\frac{g}{\eta}\right)^{2} \ll 1$. This means that we are working in the regime of the Georgi-Glashow model close to the Bogomolny-Prasad-Sommerfield limit.

Note further that in the limit $g \gg \sqrt{m}$, the dilute gas approximation holds perfectly for monopole plasma. Indeed, this approximation implies that the mean distance between monopoles, equal to $\zeta^{-1 / 3}$, should be much larger than the inverse mass of the $W$-boson. By virtue of eq. (3) and the fact that the function $\epsilon$ is of the order of unity, we obtain that this requirement is equivalent to the following one:

$$
\sqrt{\frac{\eta}{g}} \delta\left(\frac{\lambda}{g^{2}}\right) \mathrm{e}^{-4 \pi \eta / g} \ll 1 .
$$

Although at $\lambda \ll g^{2}$ the function $\delta$ grows, the speed of this growth is sp that at $g \ll \eta$, the l.h.s. of the inequality (6) remains exponentially small $\mathrm{l}$. Another consequence of this fact is that in the regime of the Georgi-Glashow model under discussion, the Debye mass of the dual photon, $m_{D}$, remains

contribution: submitted to World Scientific on December 28, 2013 
exponentially small as well. In particular, the inequality $m_{D} \ll m$, under which the full mass $M$ was derived, holds due to this smallness. Also, due to the same reason, the mean field approximation, under which the effective field theory (1) is applicable, remains valid as well with the exponential accuracy. This approximation means that one can disregard the fluctuations of individual monopoles only provided that in the Debye volume $m_{D}^{-3}$ there contained a lot of them. This condition can formally be written as

$$
\left[\text { average density }=\frac{\partial \ln \mathcal{Z}}{V \partial \ln \zeta} \simeq 2 \zeta\right] \times m_{D}^{-3} \gg 1
$$

where $V$ is the 3D volume of observation. This yields the inequality $g^{3} \gg \zeta$, which is really satisfied owing to the above-discussed exponential smallness of fugacity $\zeta$.

\section{The RG flow}

At finite temperature $T \equiv 1 / \beta$, one should supply all the fields in the model (11) with the periodic boundary conditions in the time direction, with the period equal to $\beta$. The lines of the magnetic field of a monopole thus cannot cross the boundary of the one-period region in the time direction and should go parallel to this boundary at the distances larger than $\beta$. Therefore, monopoles separated by such distances interact via the $2 \mathrm{D}$ Coulomb potential, rather than the 3D one. Recalling that the average distance between monopoles is equal to $\zeta^{-1 / 3}$, we conclude that at $T \geq \zeta^{1 / 3}$, the monopole ensemble becomes two-dimensional.

This result can also be obtained formally by computing the following sum over Matsubara frequencies:

$$
\frac{1}{|\mathbf{x}|} \equiv \sum_{n=-\infty}^{+\infty} \frac{1}{\sqrt{\vec{x}^{2}+(\beta n)^{2}}}=2 T \sum_{n=-\infty}^{+\infty} K_{0}(2 \pi T|\vec{x}| n) \simeq-2 T \ln (\mu|\vec{x}|) .
$$

Here, $\mu$ denotes the IR momentum cutoff, $\vec{x} \equiv\left(x^{1}, x^{2}\right)$, and without the loss of generality we have considered the case $x_{0}=0$. Next, $K_{0}$ stands for the modified Bessel function, which is rapidly decreasing. Owing to this fact, the term with $n=0$ (the so-called zero Matsubara mode) dominates in the whole sum, which yields the last equality. In the same way, we obtain for the Yukawa propagator:

contribution: submitted to World Scientific on December 28, 2013 


$$
\begin{gathered}
\frac{\mathrm{e}^{-m|\mathbf{x}|}}{|\mathbf{x}|} \equiv \sum_{n=-\infty}^{+\infty} \frac{\mathrm{e}^{-m \sqrt{\vec{x}^{2}+(\beta n)^{2}}}}{\sqrt{\vec{x}^{2}+(\beta n)^{2}}}= \\
=2 T \sum_{n=-\infty}^{+\infty} K_{0}\left(m|\vec{x}| \sqrt{1+\left(\frac{2 \pi T n}{m}\right)^{2}}\right) \simeq 2 T K_{0}(m|\vec{x}|) .
\end{gathered}
$$

These equations mean that the strength of the monopole-antimonopole interaction, stemming from eq. (2) at finite temperature, is proportional itself to the temperature. Owing to this fact, at low temperatures the interaction is weak, i.e. monopoles exist in the plasma phase, whereas at the temperatures higher than some critical one, $T_{c}$, they form monopole-antimonopole molecules. This situation is reversed with respect to the standard BerezinskiiKosterlitz-Thouless (BKT) phase transition in the 2D XY model $\mathrm{E}$. There, the strength of the interaction is $T$-independent, which leads to the molecular phase at low temperatures and to the plasma phase at high temperatures. In our model, the critical temperature of the phase transition is then the one, below which the mean squared separation of a monopole and an antimonopole in the molecule diverges. According to the formulae (7) and (8), this separation reads

$$
\left\langle L^{2}\right\rangle \propto \int d^{2} x|\vec{x}|^{2-\frac{8 \pi T}{g^{2}}} \exp \left[\frac{4 \pi T}{g^{2}} K_{0}(m|\vec{x}|)\right] .
$$

This yields $T_{c}=g^{2} /(2 \pi)$, which coincides with the result obtained in ref. 目 without accounting for the Higgs field. Clearly, $T_{c} \gg \zeta^{1 / 3}$, which means that there exists a broad range of temperatures where the monopole ensemble (plasma) is two-dimensional.

Let us now proceed with the formal RG analysis of the leading $\left(m_{D} / m\right)$ part of the action (5), which has been performed in ref. t. This part of the action can be written as

$$
\begin{gathered}
S=-\int d^{3} x \times \\
\times\left[\frac{1}{2}\left(\partial_{x}^{2}+\partial_{y}^{2}+a_{1} \partial_{t}^{2}\right) \chi+2 \zeta a_{2} \cos \left(g_{m} \chi\right)+\left(\frac{g_{m} \zeta a_{3}}{m}\right)^{2} \cos \left(2 g_{m} \chi\right)\right],
\end{gathered}
$$

contribution: submitted to World Scientific on December 28, 2013 
where $a_{1}=a_{2}=a_{3}=1$. The idea of derivation of the RG equations 10 is to split the cutoff field into two pieces, $\chi_{\Lambda}=\chi_{\Lambda^{\prime}}+h$. Here, the field $h$ includes the modes with the momenta lying in the range between $\Lambda^{\prime}$ and $\Lambda$ and all possible Matsubara frequencies. After that, the field $h$ should be integrated out, which yields the same action, but with another values of parameters $a_{i}$ 's. The RG equations can then be derived by performing the infinitesimal transformation $\Lambda^{\prime}=\Lambda-\delta \Lambda$ and comparing the deviation of the values of $a_{i}{ }^{\prime}$ 's from unity. In this way, we arrive at the following system of RG equations (see ref. 1 for some details of the derivation):

$$
\begin{gathered}
d x=-x^{3} z^{2} d t, \quad d z^{2}=-2 z^{2}\left(\pi x \frac{\tau}{2} \operatorname{coth} \frac{\tau}{2}-2\right) d t, \\
d \ln u=\left(\frac{3}{4}-\pi x \frac{\tau}{2} \operatorname{coth} \frac{\tau}{2}\right) d t, \quad d \ln \tau=-2 d t .
\end{gathered}
$$

Here, $t=\ln (T / \Lambda), x=T g_{m}^{2} /\left(4 \pi^{2}\right), u=\Lambda^{-3 / 4} \sqrt{g_{m} \zeta m^{-1}}, \tau=A \Lambda / T, z=$ $B \zeta /\left(\Lambda^{2} T\right)$ with $A$ and $B$ standing for some inessential constants.

The above-presented equation for the dimensionless $m$-dependent parameter $u$ is a new one with respect to the other equations. Those can be derived independently 10 in the limit when the Debye mass of the dual photon is considered to be negligibly small with respect to the Higgs one. In the limit $t \rightarrow \infty$ (or, equivalently, $\tau \rightarrow 0$ ) the RG flow for the quantities $x$ and $z$ becomes that of the $2 \mathrm{D} \mathrm{XY}$ model with the $T$-dependent strength of the interaction. In particular, we straightforwardly obtain the BKT phase transition point $z_{c}=0, x_{c}=2 / \pi$, which reproduces the critical temperature $T_{c}$, derived above heuristically. In the vicinity of the BKT transition point, the $\mathrm{RG}$ trajectories stemming from the integration of the equations for $x$ and $z$ are typical hyperbolae of the XY model. They are defined by the equation $\left(x-x_{c}\right)^{2}-(2 / \pi)^{4} z^{2}=$ const.

In the same XY-model limit, the RG equation for $u$ becomes remarkably simple, $d \ln u=\left(\frac{3}{4}-\pi x\right) d t$, and can be integrated along the separatrices $T=T_{c}$, which yields

$$
\ln u=-\frac{5}{2 \pi\left(x-x_{c}\right)}+\frac{7}{4} \ln \frac{2 x}{\pi\left|x-x_{c}\right|}+\frac{3}{2 \pi^{2} x^{2}}-\frac{1}{\pi x}+\text { const. }
$$

This equation means that at $x \rightarrow x_{c}+0, u$ exponentially vanishes along the respective (right) separatrix, whereas at $x \rightarrow x_{c}-0, u$ exponentially grows along the left separatrix. By virtue of eq. (3) with $\epsilon \sim 1$, the first of these facts can formally be written as

contribution: submitted to World Scientific on December 28, 2013 


$$
\frac{\eta}{g} \delta^{2}\left(\frac{\lambda}{g^{2}}\right) \mathrm{e}^{-8 \pi \eta / g} \ll\left(\frac{m}{g^{2}}\right)^{2}\left(\frac{\Lambda}{\eta^{2}}\right)^{3},
$$

and the second fact corresponds to the change of the symbol "«" to the opposite one. Clearly, varying two large parameters $(\eta / g)$ and $\left(g^{2} / m\right)$, as well as the ratio $\left(\Lambda / \eta^{2}\right)$, we may occur in the region of validity of any of these two inequalities. In another words, independently along which of the two separatrices the theory approaches its critical point, we can adjust the parameters $(\eta / g)$ and $\left(\Lambda / \eta^{2}\right)$ in such a way that the respective inequality holds at $g \gg \sqrt{m}$. Thus, we conclude that the theory (9) reduced by the RG flow to the 2D XY model stays within the original approximation $g \gg \sqrt{m}$ when the flow drives this theory in the vicinity of the critical point along the separatrices $T=T_{c}$.

\section{Acknowledgments}

The author is grateful to Prof. A. Di Giacomo for useful discussions and to Dr. N. Agasian, in collaboration with whom the paper $t$ has been written. This work has been supported by INFN and partially by the INTAS grant Open Call 2000, project No. 110. And last but not least, the author acknowledges the organizers of the Tenth Lomonosov Conference on Elementary Particle Physics (Moscow, 23-29 August, 2001) for an opportunity to present the above-discussed results in a very stimulating atmosphere.

\section{References}

1. A.M. Polyakov, Nucl. Phys. B 120, 429 (1977).

2. N.O. Agasian and K. Zarembo, Phys. Rev. D 57, 2475 (1998).

3. G. Dunne, I.I. Kogan, A. Kovner, and B. Tekin, JHEP 01, 032 (2001).

4. N. Agasian and D. Antonov, JHEP 06, 058 (2001).

5. K. Dietz and Th. Filk, Nucl. Phys. B 164, 536 (1980).

6. M.K. Prasad and C.M. Sommerfield, Phys. Rev. Lett. 35, 760 (1975);

E.B. Bogomolny, Sov. J. Nucl. Phys. 24, 449 (1976).

7. T.W. Kirkman and C.K. Zachos, Phys. Rev. D 24, 999 (1981).

8. V.G. Kiselev and K.G. Selivanov, Phys. Lett. B 213, 165 (1988).

9. V.L. Berezinskii, Sov. Phys.- JETP 32, 493 (1971); J.M. Kosterlitz and D.J. Thouless, J. Phys. C 6, 1181 (1973); J.M. Kosterlitz, J. Phys. C 7, 1046 (1974).

10. B. Svetitsky and L.G. Yaffe, Nucl. Phys. B 210, 423 (1982).

contribution: submitted to World Scientific on December 28, 2013 